\title{
Medial auditory thalamic stimulation as a conditioned stimulus for eyeblink conditioning in rats
}

\author{
Matthew M. Campolattaro, Hunter E. Halverson, and John H. Freeman ${ }^{1}$ \\ Department of Psychology, University of lowa, lowa City, lowa 52242, USA
}

\begin{abstract}
The neural pathways that convey conditioned stimulus (CS) information to the cerebellum during eyeblink conditioning have not been fully delineated. It is well established that pontine mossy fiber inputs to the cerebellum convey CS-related stimulation for different sensory modalities (e.g., auditory, visual, tactile). Less is known about the sources of sensory input to the pons that are important for eyeblink conditioning. The first experiment of the current study was designed to determine whether electrical stimulation of the medial auditory thalamic nuclei is a sufficient CS for establishing eyeblink conditioning in rats. The second experiment used anterograde and retrograde tract tracing techniques to assess neuroanatomical connections between the medial auditory thalamus and pontine nuclei. Stimulation of the medial auditory thalamus was a very effective CS for eyeblink conditioning in rats, and the medial auditory thalamus has direct ipsilateral projections to the pontine nuclei. The results suggest that the medial auditory thalamic nuclei and their projections to the pontine nuclei are components of the auditory CS pathway in eyeblink conditioning.
\end{abstract}

A primary emphasis in neurobiological analyses of Pavlovian eyeblink conditioning has been on identifying the anatomical sites and cellular mechanisms of memory storage (Christian and Thompson 2003; Thompson 2005). Less emphasis has been placed on identifying the neural pathways that conduct stimulation from conditioned stimuli to the sites of memory induction and retention. The cerebellum is the anatomical site of memory storage in Pavlovian eyeblink (eyelid and nictitating membrane movement) conditioning (Christian and Thompson 2003; Thompson 2005; Ohyama et al. 2006). Cerebellar function may be influenced by processes occurring within its sensory input pathways, interactions among components of the input pathways, and sources of feedback to the input pathways (Clark et al. 1997; Bao et al. 2000; Medina et al. 2002). A full characterization of the sensory input pathways to the cerebellum necessary for motor learning is, therefore, critical for developing a comprehensive understanding of cerebellar function. Identification of sensory input pathways to the cerebellum is also critical for elucidating the mechanisms underlying the ontogeny of motor learning (Freeman et al. 2005).

Several key components of the conditioned stimulus (CS) pathway in eyeblink conditioning have been identified using lesion, inactivation, unit recording, stimulation, and tract tracing techniques (Steinmetz et al. 1986, 1987, 1989; Lewis et al. 1987; Knowlton and Thompson 1988; Steinmetz 1990; Steinmetz and Sengelaub 1992; Gould et al. 1993; Tracy et al. 1998; Hesslow et al. 1999; Bao et al. 2000; Freeman and Rabinak 2004; Freeman et al. 2005). The pontine mossy fiber projection to the cerebellum is necessary for eyeblink conditioning with CSs of different modalities and stimulation of the pontine nuclei or the middle cerebellar peduncle is sufficient for eyeblink conditioning in different species (Steinmetz et al. 1986, 1989; Steinmetz 1990; Tracy et al. 1998; Hesslow et al. 1999; Freeman and Rabinak 2004; Freeman et al. 2005). For an auditory CS, there is a monosynaptic projection from the cochlear nuclei to the pontine nuclei (Steinmetz et

\footnotetext{
'Corresponding author.
}

E-mail john-freeman@uiowa.edu; fax (319) 335-0191.

Article is online at http://www.learnmem.org/cgi/doi/10.1101//m.465507. al. 1987; Steinmetz and Sengalaub 1992; Gould et al. 1993). The monosynaptic cochlear-pontine projection may play an important role in auditory eyeblink conditioning, but a recent study found that auditory structures that receive direct or indirect input from the cochlear nuclei also play an important role in eyeblink conditioning. Halverson and Freeman (2006) found that unilateral lesions of the medial auditory thalamic nuclei (MATN), including the medial nucleus of the medial geniculate, posterior intralaminar nucleus, and suprageniculate contralateral to the conditioned eye severely impaired acquisition of eyeblink conditioning using a tone CS in rats. The findings of Halverson and Freeman (2006) suggested that the contralateral medial auditory thalamus could play an essential role in auditory eyeblink conditioning. A major unresolved issue from this study was how the medial auditory thalamus might influence the eyeblink conditioning circuitry. The most straightforward way for the MATN to play a role in the CS pathway would be through an axonal projection to the pontine mossy fiber system.

The first experiment of the current study was designed to determine whether electrical stimulation of the MATN is sufficient for establishing associative eyeblink conditioning in rats. Rats were given stimulation of the medial auditory thalamus as a CS paired with a peripheral shock unconditioned stimulus (US). A control group was given unpaired presentations of medial auditory thalamic stimulation and the US. Experiment 2 was designed to determine whether the MATN send axonal projections to the pontine nuclei. Anterograde and retrograde tracing methods were used to assess the projections of the medial auditory thalamus in untrained rats.

\section{Results}

\section{Experiment 1: Stimulation of the MATN as a CS} is sufficient for eyeblink conditioning

Experiment 1 examined whether electrical stimulation of the medial auditory thalamus is sufficient for establishing associative eyeblink conditioning in rats. Rats were implanted with a bipolar stimulating electrode in the medial nucleus of the medial genicu- 
late. They were then given paired or unpaired training with medial auditory thalamic stimulation $(300 \mathrm{msec}, 200 \mathrm{~Hz}$ train; 100 usec biphasic pulses; $30-100 \mu \mathrm{A}$ ) as the CS and a periorbital shock US (10 msec, 1.0-2.0 mA). Electrodes placed in the medial nucleus of the medial geniculate could possibly affect eyeblink conditioning by stimulating nearby structures, including the anterior pretectal nucleus medially and the ventral nucleus of the medial geniculate laterally, if the current spread beyond the target. A group of rats were given intentionally misplaced electrodes in the anterior pretectal nucleus or ventral nucleus of the medial geniculate to determine whether stimulation of these structures produces conditioning that is as rapid as the conditioning observed with electrodes placed in the medial auditory thalamus. Rapid conditioning in the rats with misplaced electrodes would cast doubt upon the specificity of the medial auditory thalamic stimulation.

The MATN receive input from the ipsilateral inferior colliculus but also send projections back to it (see experiment 2). It would, therefore, be possible that stimulation of the medial auditory thalamus has its effects on eyeblink conditioning through activation of the inferior colliculus rather than through activation of a direct projection to the pontine nuclei. To assess the possible contribution of the thalamic projection to the inferior colliculus in eyeblink conditioning with medial auditory thalamic stimulation as the CS, a group of rats was given electrolytic lesions of the inferior colliculus ipsilateral to the stimulation site 1 wk before the onset of paired eyeblink conditioning.

\section{Eyeblink conditioning}

Rats in the control group that received paired training with MATN stimulation acquired conditioning very rapidly, whereas control rats that received unpaired training with MATN stimulation did not (Fig. 1). Rats with intentionally missed electrodes in the anterior pretectal nucleus or the ventral medial geniculate showed only a small increase in conditioned response (CR) percentage during the third training session (Fig. 1). The rats given lesions of the inferior colliculus showed conditioning that was comparable to the paired control group (Fig. 1).

The effects described above were confirmed by an ANOVA that revealed a significant effect of the group factor, $F_{(3,13)}=67.15, P<0.001$. Post hoc tests showed that the control

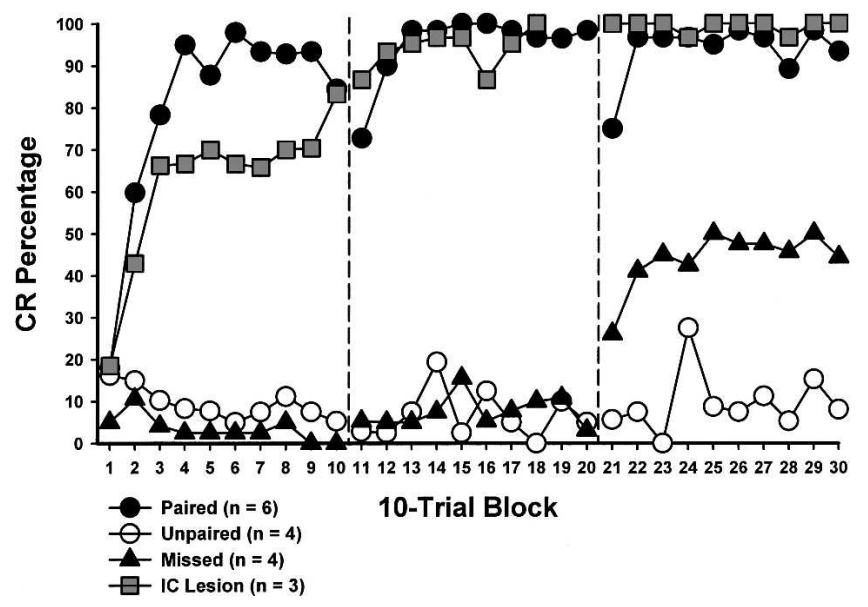

Figure 1. Medial auditory thalamic stimulation as a conditioned stimulus supports associative eyeblink conditioning. Mean conditioned response (CR) percentage for rats with accurately placed electrodes given paired (black circles) or unpaired training (white circles), rats given intentionally misplaced electrodes (black triangles), and rats given inferior colliculus lesions (gray squares) across 30 10-trial blocks of trials. The vertical dashed lines indicate breaks between daily training sessions.

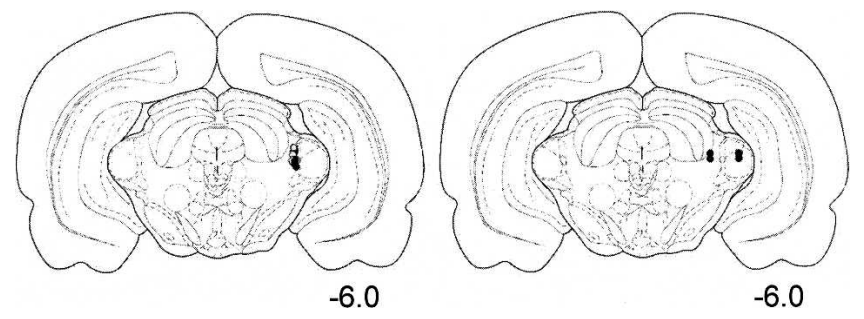

Figure 2. Electrode placements. The tips of stimulation electrodes in the control rats given paired (black circles) or unpaired (gray circles) training were in or within $0.2 \mathrm{~mm}$ of the medial division of the medial geniculate (left). Rats given intentionally misplaced electrodes had tip placements $1.0 \mathrm{~mm}$ medial and lateral to the medial division of the medial geniculate (right). Numbers indicate the anterior-posterior stereotaxic coordinates of the sections relative to bregma (This figure was reproduced with permission from Elsevier $\odot$ 1998, Paxinos and Watson 1998).

and lesion paired groups produced a greater CR percentage than the groups given unpaired training and paired training with misplaced electrodes, $P<0.01$. Two of the rats with intentionally misplaced stimulation electrodes on the medial side of the MATN (Fig. 2) acquired conditioning, albeit slower than rats with accurately placed electrodes. The two rats with intentionally misplaced stimulation electrodes in the ventral medial geniculate (Fig. 2) did not show an increase in CRs across training. These rats received an additional session of paired training with the stimulation CS followed by three sessions of paired training with a tone CS (300 msec, $2.0 \mathrm{kHz}, 85 \mathrm{~dB})$. The rats with missed electrodes that did not condition with the stimulation CS were given training with a tone CS to determine whether damage to the MGN caused by the electrode impaired conditioning. These rats acquired conditioning with the tone CS during the three sessions of training (mean CR percentage across sessions $=15.5 \%, 38.6 \%$, and $83.7 \%$, respectively). An additional session of paired training with stimulation was then given to assess whether any behavioral savings could be observed with stimulation after learning with a peripheral tone CS. However, these rats showed very few CRs during this session (mean $=6.6 \%$ ). The results of the follow up training and testing sessions indicated that the electrode placements in the ventral medial geniculate were not effective for producing conditioning, suggesting that the thalmo-cortical projection does not play a significant role in delay conditioning.

Conditioned response amplitude was measured on CS-alone trials. Only the control and lesion groups given paired training produced enough CRs during CS-alone trials for meaningful statistical analyses. An ANOVA of the CR amplitude data found no significant group differences between the paired control and lesion groups. Both groups showed a progressive increase in CR amplitude across training (mean $=3.43,4.65,5.44$, respectively). No significant group differences were found for the CR onset or peak latency measures, which both decreased across sessions (onset mean in $\mathrm{msec}=198.47,128.49,123.75$; peak mean in $\mathrm{msec}=296.10,255.05,253.87)$.

\section{Stimulation electrode placement}

The electrodes in the paired and unpaired control groups were consistently placed in or within $0.2 \mathrm{~mm}$ of the medial nucleus of the medial geniculate (Fig. 2, left). The paired and unpaired controls had equivalent electrode placements. The rats given inferior colliculus lesions also had electrodes placed within $0.2 \mathrm{~mm}$ of the medial nucleus of the medial geniculate. Intentionally missed electrodes were placed in the ventral nucleus of the medial geniculate and in the anterior pretectal nucleus (Fig. 2, right). 


\section{Inferior colliculus lesions}

The inferior colliculus contralateral to the conditioned eye was damaged extensively. Figure 3 depicts the largest and smallest inferior colliculus lesions. The lesions were large but restricted to the inferior colliculus. The most extensive damage was in anterior nuclei, which is where most of the labeled axon terminals were found following biotinylated dextran amine (BDA) injections into the MATN (see Experiment 2, below).

\section{Experiment 2: MATN project to the pontine nuclei}

The results of Experiment 1 indicate that stimulation of the MATN can serve as a sufficient CS for eyeblink conditioning. If the MATN are part of the auditory CS pathway, they should send axonal projections to the pontine nuclei either directly or indirectly. Anterograde and retrograde tract tracing were used in Experiment 2 to determine whether there are direct projections from the MATN to the pontine nuclei. BDA was used as an anterograde tracer to determine whether the MATN send axon terminals to the pontine nuclei. Fluorogold (FG) injected into the basal pontine nuclei was used as a retrograde tracer.

The injection sites for BDA included the medial nucleus of the medial geniculate, dorsal posterior intralaminar nucleus, and suprageniculate nucleus (Fig. 4A). Some of the BDA was taken up by cells in the visual nuclei that are dorsal to the medial geniculate. The injection sites for FG included the dorsolateral, lateral, and ventral pontine nuclei (Fig. 5A).

BDA-labeled axon terminals were evident in the dorsolateral, lateral, medial, and ventral pontine nuclei ipsilateral to the injection site (Fig. 4C,D). No terminals were observed in the contralateral pontine nuclei. The terminals appeared to have arisen from descending fiber projections through the area of the ipsilateral lateral lemniscus and the dorsal pons. The same pattern of terminal labeling in the pontine nuclei was seen in all of the rats given BDA infusions.

Retrogradely labeled neurons were seen in the medial division of the medial geniculate, the most dorsal part of the poste-

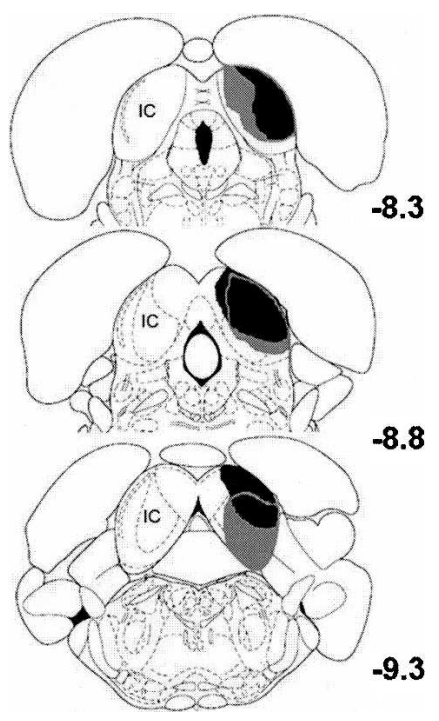

Figure 3. Inferior colliculus (IC) lesions. The largest (gray shaded areas) and smallest (black shaded areas) unilateral lesions of the IC. The lesions were ipsilateral to the MATN stimulation electrode (contralateral to the conditioned eye). Numbers indicate the anterior-posterior stereotaxic coordinates of the sections relative to bregma (This figure was reproduced with permission from Elsevier (C) 1998, Paxinos and Watson 1998).
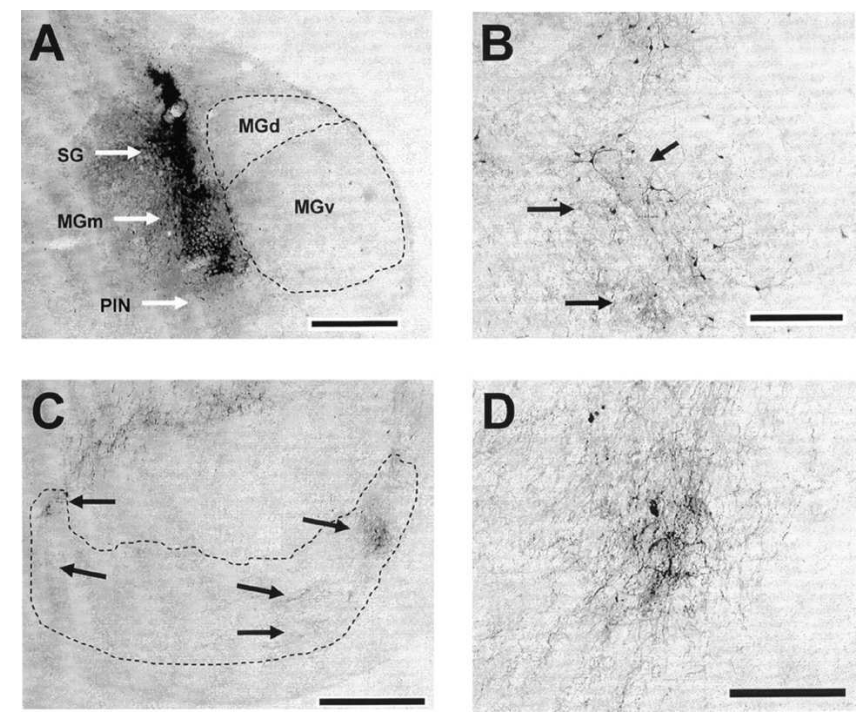

Figure 4. BDA labeling in the pontine nuclei and inferior colliculus following injection into the medial auditory thalamus. $(A)$ Representative BDA injection site (arrows) within the suprageniculate (SG), medial nucleus of the medial geniculate $(\mathrm{MGm})$, and posterior intralaminar nucleus (PIN). (MGd) dorsal medial geniculate; (MGv) ventral medial geniculate. Scale bar, $500 \mu \mathrm{m}$. (B) Anterograde (arrows) and retrograde labeling in the ipsilateral anterior external inferior colliculus. Scale bar, $250 \mu \mathrm{m}$. (C) Anterograde labeling (arrows) within the ipsilateral basa pontine nuclei, which are outlined by the dashed line. Scale bar, $500 \mu \mathrm{m}$. $(D)$ Higher magnification view of anterograde labeling in the lateral pontine nucleus. Scale bar, $125 \mu \mathrm{m}$.

rior intralaminar nucleus, posterior limitans nucleus, and throughout the suprageniculate nucleus in rats given infusions of FG into the pontine nuclei (Fig. 5B,C). The thalamic retrograde labeling was exclusively ipsilateral to the infusion site.

Labeled neurons and terminals were observed throughout the ipsilateral inferior colliculus (Figs. 4B, 6B). Labeling was denser in the anterior external inferior colliculus relative to the central nucleus. Labeled neurons were seen in the inferior colliculus with BDA (Fig. 4B) and FG (Fig. 6B). As with the pontine nuclei, the terminal labeling with BDA was ipsilateral in the inferior colliculus.

The cochlear nuclei exhibited retrogradely labeled neurons bilaterally, primarily in the ventral nucleus. The density of labeled neurons appeared to be greater in the contralateral cochlear nuclei, as seen in rabbits (Steinmetz et al. 1987) (Fig. 6A).

Extensive terminal labeling and retrograde labeling were observed in the intermediate and deep layers of the superior colliculus and the anterior pretectal nucleus (Fig. 7). A particularly dense area of labeled neurons was seen in the deep superior colliculus just dorsal to the PAG in the rats given infusions of FG into the pontine nuclei (Fig. 7A). The dorsal and lateral PAG were also strongly labeled. The PAG had a high density of retrogradely labeled cells and terminals. The PAG labeling was exclusively ipsilateral to the injection sites.

The ipsilateral cerebral cortex showed extensive retrograde labeling, as seen in previous anatomical studies (Legg et al. 1989). Labeled neurons were mostly seen in layer 5 (Fig. 6C). Sensory and motor areas were labeled heavily relative to cingulate, perirhinal, and entorhinal areas.

\section{Discussion}

Rats given electrical stimulation of the medial auditory thalamus as a CS paired with a peripheral US showed rapid acquisition of 
eyeblink conditioning. Rats given unpaired presentations of the stimulation CS and the peripheral US showed no increase in eyeblink responses across training trials; indicating that acquisition of eyeblink conditioning in the paired group was due to associative learning. Conditioned responses produced by the paired group increased in amplitude across training trials, and the peak amplitude occurred just prior to the onset of the US, as seen with conditioning in rats using peripheral CSs (Nolan and Freeman 2006). Rats with electrodes intentionally misplaced $1.0 \mathrm{~mm}$ lateral to the medial nucleus of the medial geniculate showed no conditioning. Rats with electrodes placed $1.0 \mathrm{~mm}$ medial to the medial nucleus of the medial geniculate showed acquisition of eyeblink conditioning but at a substantially lower rate than rats with accurately placed electrodes. Acquisition of eyeblink conditioning with auditory thalamic stimulation as the CS was not impaired by lesions of the inferior colliculus, suggesting that the stimulation probably did not produce conditioning through thalamic projections to the inferior colliculus.

The conditioning seen in rats given paired presentations of auditory thalamic stimulation and a peripheral US indicates that activation of the medial auditory thalamus is sufficient for establishing associative eyeblink conditioning in rats. Moreover, rats in the paired group showed a rate of acquisition that was sub-
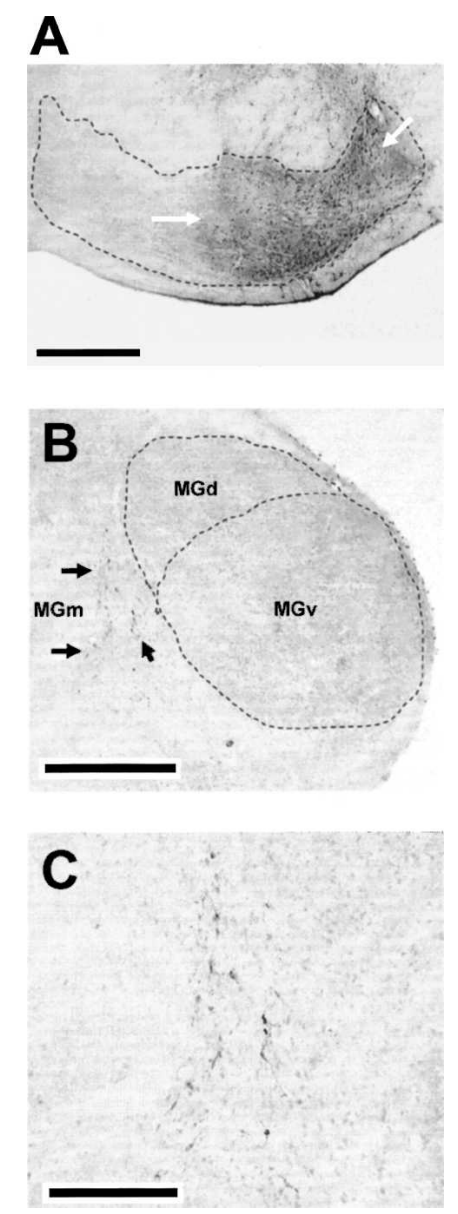

Figure 5. FG labeling in the medial auditory thalamus following injection into the lateral pontine nuclei. (A) Representative FG injection site within the pontine nuclei. Scale bar, $500 \mu \mathrm{m}$. (B) Retrograde labeling of neurons in the ipsilateral medial auditory thalamic nuclei (arrows). (MGd) indicates dorsal medial geniculate; (MGv) ventral medial geniculate. Scale bar, $500 \mu \mathrm{m}$. (C) Higher magnification view of retrograde labeling in the ipsilateral medial auditory thalamus. Scale bar, $250 \mu \mathrm{m}$.
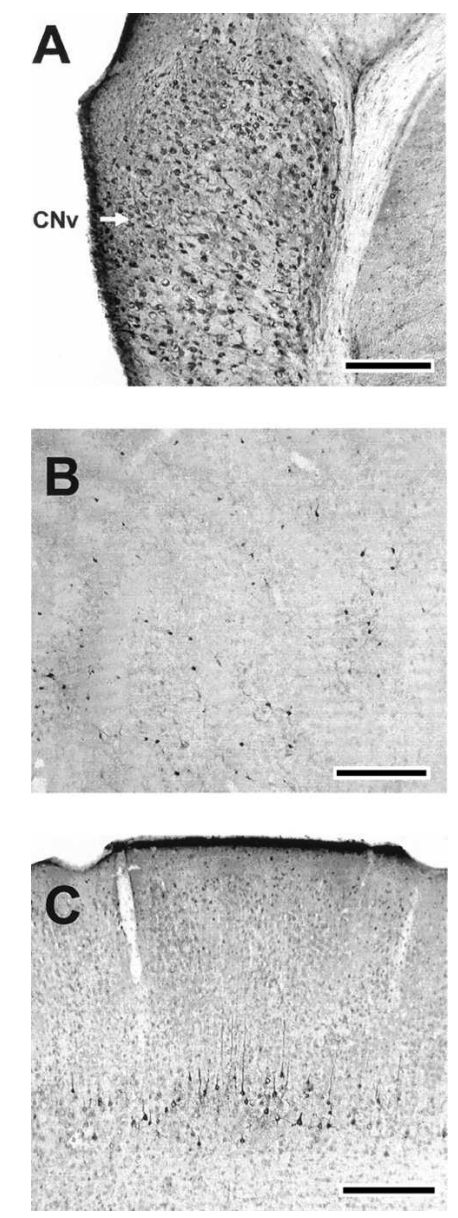

Figure 6. FG labeling in the cochlear nuclei, inferior colliculus, and cerebral cortex. $(A)$ Retrograde labeling of neurons in the contralateral ventral cochlear nucleus (arrow). (B) Retrograde labeling of neurons in the ipsilateral anterior external inferior colliculus. (C) Retrograde labeling of neurons in the ipsilateral motor cortex. Scale bars, $500 \mu \mathrm{m}$.

stantially faster than seen with tone or light CSs in our laboratory, reaching an asymptotic level of conditioning within the first 100-trial training session. Asymptotic conditioning using a peripheral CS is typically seen after 200 training trials. The rapid conditioning seen in the paired group suggests that the projection from the medial auditory thalamus to the pons is very effective and sufficient to serve as a CS when other sensory projections to the pons are not activated.

Stimulation of the ventral medial geniculate as a CS was ineffective for establishing eyeblink conditioning in the current study. The ventral nucleus of the medial geniculate provides a large proportion of the thalamic input to the primary auditory cortex (Winer 1992), which then projects directly to the pontine nuclei. Stimulation of the ventral medial geniculate or auditory cortex has been used as a CS to establish eyeblink conditioning in rabbits (Knowlton and Thompson 1992; Nowak et al. 1999). Auditory cortex stimulation as a CS resulted in robust eyeblink conditioning that was cerebellum-dependent, even though the cerebral cortex is not essential for eyeblink conditioning (Knowlton and Thompson 1992). Stimulation of the ventral medial geniculate was moderately effective as a CS when relatively strong stimulation parameters were used $(150 \mu \mathrm{A}, 200 \mathrm{~Hz}, 1.2 \mathrm{msec})$ (Nowak et al. 1999). It is possible that the stimulation parameters used in the current study (30-100 $\mu \mathrm{A}, 200 \mathrm{~Hz}, 0.1 \mathrm{msec})$ were 

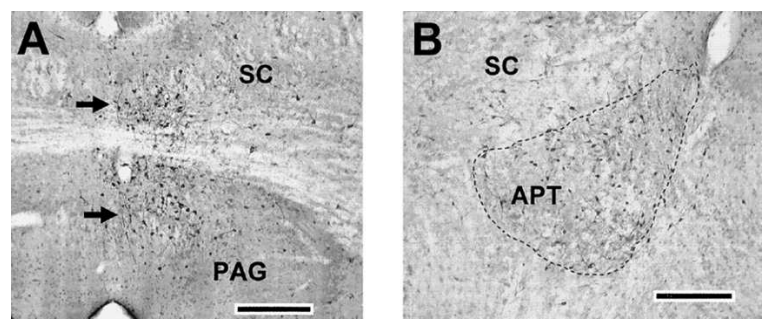

Figure 7. FG labeling in the anterior pretectal nucleus, periaqueductal gray, and superior colliculus. (A) Retrograde labeling (arrows) of neurons in the superior colliculus (SC) and periaqueductal gray (PAG). Scale bar, $250 \mu \mathrm{m}$. (B) Retrograde labeling of neurons in the anterior pretectal nucleus (APT). Scale bar, $250 \mu \mathrm{m}$.

simply insufficient for activating the thalamo-cortical auditory system. On the other hand, the parameters used by Nowak et al. (1999) may have produced conditioning through current spread to the neighboring MATN. The failure to obtain conditioning in the rats given stimulation of the ventral medial geniculate may, therefore, be consistent with the findings of the previous studies.

In contrast to the ventral medial geniculate, stimulation of the anterior pretectal nucleus as a CS produced a small amount of eyeblink conditioning. The anterior pretectal nucleus has been implicated as part of the visual CS pathway for eyeblink conditioning in rabbits (Koutalidis et al. 1988) and projects directly to the pontine nuclei (Fig. 7B). However, there are no published reports describing the use of pretectal stimulation as a CS in eyeblink conditioning. The modest conditioning seen in the rats with electrodes placed in the anterior pretectal nucleus may have been due to stimulation of its projection to the pontine nuclei. The relative insufficiency of pretectal and ventral medial geniculate stimulation as a CS in the current study suggests that the medial auditory thalamic stimulation did not produce conditioning by stimulating neighboring nuclei.

Anterograde and retrograde neural tract tracers provided evidence for direct projections from the MATN to the pontine nuclei. The projections from the medial auditory thalamus to the basal pons were distributed across the dorsolateral, lateral, medial, and ventral nuclei. It is not clear which pontine nuclei are essential for auditory CS input to the cerebellum in rats. In rabbits, the dorsolateral and lateral nuclei have been implicated as the essential nuclei for an auditory CS. Lesions of the lateral and dorsolateral pontine nuclei abolish nictitating membrane CRs to auditory but not visual CSs (Steinmetz et al. 1987). Selective lesions of the different pontine nuclei have not been performed in rat eyeblink conditioning studies, but a unit recording study of auditory CS-elicited neuronal activity in the pontine nuclei in infant rats revealed auditory responses in the dorsolateral, lateral, medial, and ventral nuclei (Freeman and Muckler 2003). The rodent auditory CS pathway may be more distributed among the pontine nuclei relative to rabbits, suggesting that each of the auditory thalamo-pontine projections observed in the current study could play a role in transmission of CS stimulation to the cerebellum.

Precise infusions of anterograde tracers into the subnuclei of the medial auditory thalamus are needed to determine whether each thalamic nucleus projects to a different pontine nucleus or if the thalamic nuclei have overlapping projections. The possibility of overlapping projections is suggested by the findings of a previous study that found that lesions of the auditory thalamus prevented acquisition of eyeblink conditioning only with complete destruction of the medial division of the medial geniculate, posterior intralaminar nucleus, suprageniculate, and the poste- rior limitans nucleus (Halverson and Freeman 2006). Lesions that spared any of the nuclei had very little effect on eyeblink conditioning. An implication of this finding is that the thalamic nuclei provide parallel input that overlaps in the region(s) of the pons that is essential for auditory eyeblink conditioning.

As noted in previous studies, a projection from the inferior colliculus to the MATN, as well as a feedback connection from the medial auditory thalamus to the inferior colliculus, was observed (LeDoux et al. 1987; Winer 1992). The inferior colliculus in turn projects to the basal pontine nuclei (Fig. 6B; Kawamura 1975). It was therefore possible that stimulation of the medial auditory thalamus produced conditioning by activating the inferior colliculus and its projection to the pons. This possibility was partially ruled out by the demonstration of unimpaired acquisition in the group given large electrolytic lesions of the inferior colliculus. The results of the current study generally suggest that auditory CS signals are conveyed to the cerebellum via a serial pathway from the inferior colliculus to the medial auditory thalamus and then to the basal pontine nuclei.

The current findings combined with the results of a previous study (Halverson and Freeman 2006) indicate that the medial auditory thalamic contribution to the CS pathway is unilateral. Lesions of the medial auditory thalamus that were contralateral to the conditioned eye severely impaired auditory eyeblink conditioning (Halverson and Freeman 2006). In the current study, stimulation of the contralateral medial auditory thalamus was sufficient for eyeblink conditioning, and the projections from the medial auditory thalamus to the pontine nuclei were exclusively ipsilateral. The projections from the inferior colliculus to the medial thalamus were also ipsilateral. These findings suggest that although there are multiple crossed projections within the mammalian auditory system, the auditory CS pathway in eyeblink conditioning includes a unilateral projection from the inferior colliculus to the medial thalamus and then to the pons. It will be important in future studies to determine whether the auditory CS pathway component of the pontine projection to the cerebellum is also unilateral.

Projections from the PAG to the pontine nuclei are an unexpected finding of the current study. The PAG is well known as an important component in the behavioral organization of fear (LeDoux et al. 1988; Fanselow 1994), but its role in eyeblink conditioning is not well characterized. Recordings of unit activity from the PAG during eyeblink conditioning in rabbits revealed stimulus-elicited and response-related activity (McCormick et al. 1983). The effects of PAG lesions or inactivation during eyeblink conditioning have not been reported. However, lesions of the amygdala slow acquisition of eyeblink conditioning (Lee and Kim 2004; Blankenship et al. 2005). It is possible that amygdala modulation of the acquisition of eyeblink conditioning is mediated through its projection to the PAG and the PAG projection to the pons.

The current findings and those of a previous study (Halverson and Freeman 2006) demonstrated that the MATN play an important role in auditory eyeblink conditioning. The medial auditory thalamus could serve as part of the sensory input that conveys tone information to the cerebellum. Another possibility is that the thalamic nuclei modulate the short latency pathway from the cochlear nuclei to the pons, providing gain to boost the auditory signal en route to the cerebellum. It is also possible that conditioning-specific modifications of thalamic activity (Disterhoft and Olds 1972; Gabriel et al. 1975; Ryugo and Weinberger 1978; Birt and Olds 1981; Supple and Kapp 1989; Edeline and Weinberger 1992; Lennartz and Weinberger 1992; McEchron et al. 1995; O'Connor et al. 1997; Maren et al. 2001; Poremba and Gabriel 2001) are necessary for this hypothetical modulatory boost of auditory input to the pons. 


\section{Materials and Methods}

\section{Experiment 1}

\section{Subjects}

Subjects were 17 adult Long Evens male rats (250-300 g), $150 \mathrm{~d}$ of age at the beginning of the experiment. The rats were housed in Spence Laboratories of the Psychology at the University of Iowa with a 12-h light-dark cycle, with light onset at 7:00 am.

\section{Groups}

Rats were assigned to groups that received paired ( $n=13)$ or unpaired $(n=4)$ training. Four of the rats in the paired group were given intentionally misplaced electrodes (two in the anterior pretectal nucleus, two in the ventral nucleus of the medial geniculate), and three were given inferior colliculus lesions.

\section{Surgery}

One week prior to training, rats were removed from their home cage and anesthetized by an i.p. injection of sodium pentobarbital $(80 \mathrm{mg} / \mathrm{kg})$. An i.p. injection of atropine sulfate $(0.45 \mathrm{mg} / \mathrm{kg})$ was administered to reduce respiratory tract secretions. After onset of anesthesia, a bipolar stimulating electrode (Plastics One, Inc.) was implanted in the right medial geniculate. The stereotaxic coordinates were $-5.7 \mathrm{~mm}$ posterior to bregma and -2.8 $\mathrm{mm}$ lateral to the midline. The bipolar stimulating electrode was lowered $-6.1 \mathrm{~mm}$ below the surface of the skull and secured to the skull with dental acrylic. Four rats were implanted with intentionally misplaced bipolar stimulating electrodes at $1.6 \mathrm{~mm}$ $(n=2)$ or $3.6 \mathrm{~mm}(n=2)$ lateral to the midline. A subset of the rats given paired training was given electrolytic lesions of the inferior colliculus ipsilateral to the stimulation electrode $(n=3)$. The lesions were produced by passing $1.0 \mathrm{~mA}$ of DC current for $10 \mathrm{sec}$ at three sites via an insect pin insulated with epoxylite. The stereotaxic coordinates were $8.3,8.8$, and $9.2 \mathrm{~mm}$ posterior to bregma; 2.2, 2.0, and $1.9 \mathrm{~mm}$ lateral to midline; and 4.2, 4.6, and $4.3 \mathrm{~mm}$ below the skull surface. Skull holes were sealed with bone wax.

All of the rats were implanted with differential electromyographic (EMG) electrodes in the left upper eyelid muscle (orbicularis oculi), and a ground electrode was attached to a stainless steel skull screw. The EMG electrode leads terminated in gold pins held in a plastic connector, which was secured to the skull with dental acrylic. A bipolar stimulating electrode (Plastics One, Inc.) for delivering the shock US was implanted in a plastic connector immediately caudal to the left eye. The bipolar electrode terminated in a plastic connector that was secured to the skull with dental acrylic.

\section{Conditioning apparatus}

The conditioning apparatus consisted of a small-animal sound attenuation chamber (BRS/LVE) with a small-animal operant chamber (BRS/LVE) contained inside. The rats were kept in the operant chamber during conditioning. Cables with connectors for the EMG, US, and CS electrodes were attached to a commutator. The electrode leads from the rat's head stage were connected to peripheral equipment and a desktop computer. Computer software controlled the delivery of stimuli and the recording of eyelid EMG activity (JSA Designs). The US was delivered through a stimulus isolator (World Precision Instruments). EMG activity was recorded differentially, filtered $(500-5000 \mathrm{~Hz})$, amplified $(2000 \times)$, and integrated (time constant $=20 \mathrm{msec}$ ). MGN stimulation was triggered through a programmable stimulator (Master 8, A.M.P.I.), which controlled signal input to the stimulus isolator (World Precision Instruments) that delivered the electrical stimulation.

\section{MATN stimulation}

Electrical stimulation of the medial auditory thalamus functioned as the CS, which was administered in a $200-\mathrm{Hz}$ train of $0.1-\mathrm{msec}$ biphasic pulses for $300 \mathrm{msec}$. The stimulation threshold for the CS was found before training by setting the stimulating current to elicit an observable behavioral response and then decreasing the current in 5- $\mu \mathrm{A}$ increments until no movement was detected. Observable movements included, but were not limited to eye blinks, orienting responses, ear movements, and head movements. A maximum stimulation threshold was set at 100 $\mu \mathrm{A}$ (range $=30-100 \mu \mathrm{A}$ ). The most typical behavioral response elicited by stimulation was a discrete movement of the left ear.

\section{Paired training}

Rats were given three paired training sessions. The paired training sessions consisted of 100 trials; each with 90 trials of the stimulation CS paired with the shock US (10 msec, $1.0-2.0 \mathrm{~mA})$ and 10 stimulation CS-alone trials, occurring on every 10th trial. The CS-alone trials were included in order to assess behavioral responses (integrated EMG activity) uncontaminated by unconditioned responses (URs). The interstimulus interval for paired trials was $290 \mathrm{msec}$. Trials were separated by an intertrial interval (ITI) that averaged $30 \mathrm{sec}$. Behavioral data were examined from computer records of EMG responses. CRs were defined as responses that crossed a threshold of $0.4 \mathrm{~V}$ above the baseline activity during the CS period, but at least $80 \mathrm{msec}$ after CS onset, to avoid contamination of the CR measures by the startle (alpha) response. The amplitude, onset latency, and peak latency of the CR were measured on CS-alone trials in which a CR occurred.

\section{Unpaired training}

Rats in the unpaired control group were given three 200-trial sessions of explicitly unpaired presentations of the CS and US. The same time durations for the CS and US were used as in the paired procedure. The ITI was set to average $15 \mathrm{sec}$ to match the total time spent in the conditioning chamber and the temporal distribution of CS and US presentations with the paired groups. The method for defining CRs was the same as that used in the paired procedure.

\section{Histology}

After training was completed, rats were euthanized with a lethal injection of sodium pentobarbital $(90 \mathrm{mg} / \mathrm{kg})$ and transcardially perfused with $0.1 \mathrm{M}$ buffered physiological saline, followed by a $10 \%$ formalin solution. The brains were post-fixed in formalin for $2 \mathrm{~d}$ and then put in a solution of 30\% sucrose in PBS before sectioning. The brains were sectioned at $50 \mu \mathrm{m}$ with a sliding microtome. Sections were then stained with thionin. The bipolar stimulating electrode location and extent of the inferior colliculus lesions was determined by examining consecutive sections.

\section{Experiment 2}

\section{Subjects}

Subjects were seven adult Long Evens male rats (250-300 g), 150 $\mathrm{d}$ old at the beginning of the experiment. The rats were housed in Spence Laboratories of the Psychology at the University of Iowa with a 12-h light-dark cycle, with light onset at 7:00 am.

\section{Surgery}

The rats were removed from their home cage and anesthetized by an i.p. injection of sodium pentobarbital $(80 \mathrm{mg} / \mathrm{kg})$. An i.p. injection of atropine sulfate $(0.45 \mathrm{mg} / \mathrm{kg})$ was administered to reduce respiratory tract secretions. After onset of anesthesia, pressure injections of $0.05 \mu \mathrm{L}$ of $4 \%$ FG were made into the lateral pontine nuclei $(n=4)$ or $0.05 \mu \mathrm{L}$ of a $10 \%$ BDA solution (Molecular Probes) into the medial division of the medial geniculate $(n=3)$. BDA and two of the FG infusions were delivered at a rate of $1.0 \mu \mathrm{L} / \mathrm{h}$ through a 30 -gauge cannula. Two of the FG injections were made through a glass pipette with a tip diameter of $70 \mu \mathrm{m}$. The cannula or glass pipette remained in position for $15 \mathrm{~min}$ before removal. The post-injection survival period was $14 \mathrm{~d}$.

\section{Anterograde labeling with BDA}

Two weeks following BDA infusion, the rats were perfused with 0.1 M PBS followed by $4 \%$ paraformaldehyde. After a postfixation period of $24 \mathrm{~h}$, the brains were sectioned on a freezing 
microtome at $50 \mu \mathrm{m}$. Visualization of BDA was accomplished by using the standard avidin-biotin complex (ABC) method (Vectastain ABC Elite Kit, Vector Laboratories). The tissue slices were incubated in the $\mathrm{ABC}$ solution for $60 \mathrm{~min}$ at room temperature. Following three washes in phosphate buffer, the tissue was incubated in $0.05 \%$ diaminobenzidine in phosphate buffer with hydrogen peroxide $(0.01 \%)$ added to the solution. The tissue was incubated until a clear reaction developed $(\sim 15 \mathrm{~min})$. The labeled sections were examined using a light microscope.

\section{Retrograde labeling with FG}

At the end of the survival period, the rats were perfused with 0.1 M PBS followed by 4\% paraformaldehyde. After a post-fixation period of $24 \mathrm{~h}$, the brains were sectioned on a freezing microtome at $50 \mu \mathrm{m}$. Visualization of FG was accomplished by immunohistochemistry with free floating sections. The brain sections were pretreated with $\mathrm{H}_{2} \mathrm{O}_{2}$ to block endogenous peroxidase, followed by incubation in normal goat serum. They were then incubated in a rabbit primary antibody for $72 \mathrm{~h}$ at $4^{\circ} \mathrm{C}$ followed by incubation in a biotinylated goat anti-rabbit secondary antibody for 90 min at room temperature. The biotinylated secondary antibody was bound to a standard $\mathrm{ABC}$ (Vectastain $\mathrm{ABC}$ Elite Kit, Vector Laboratories). The tissue slices were incubated in the $\mathrm{ABC}$ solution for $60 \mathrm{~min}$ at room temperature. Following three washes in phosphate buffer, the tissue was incubated in $0.05 \%$ diaminobenzidine in phosphate buffer with hydrogen peroxide $(0.01 \%)$ added to the solution. The tissue was incubated until a clear reaction developed ( $\sim 5 \mathrm{~min})$. The labeled sections were examined using a light microscope.

\section{Acknowledgments}

This work was supported by grants from the NIMH (MH065483) and NINDS (NS038890).

\section{References}

Bao, S., Chen, L., and Thompson, R.F. 2000. Learning- and cerebellum-dependent neuronal activity in the lateral pontine nucleus. Behav. Neurosci. 114: 254-261.

Birt, D. and Olds, M. 1981. Associative response changes in lateral midbrain tegmentum and medial geniculate during differential appetitive conditioning. J. Neurophysiol. 46: 1039-1055.

Blankenship, M.R., Huckfeldt, R., Steinmetz, J.J., and Steinmetz, J.E. 2005. The effects of amygdala lesions on hippocampal activity and classical eyeblink conditioning in rats. Brain Res. 1035: 120-130.

Christian, K.M. and Thompson, R.F. 2003. Neural substrates of eyeblink conditioning: acquisition and retention. Learn. Mem. 10: 427-455.

Clark, R.E., Gohl, E.B., and Lavond, D.G. 1997. The learning-related activity that develops in the pontine nuclei during classical eye-blink conditioning is dependent on the interpositus nucleus. Learn. Mem. 3: 532-544.

Disterhoft, J.F. and Olds, J. 1972. Differential development of conditioned unit changes in thalamus and cortex of rat. $J$. Neurophysiol. 35: 665-679.

Edeline, J.M. and Weinberger, N.M. 1992. Associative retuning in the thalamic source of input to the amygdala and auditory cortex: Receptive field plasticity in the medial division of the medial geniculate body. Behav. Neurosci. 106: 81-105.

Fanselow, M.S. 1994. Neural organization of the defensive behavior system responsible for fear. Psychonom. Bull. Rev. 1: 429-438.

Freeman Jr., J.H. and Muckler, A.S. 2003. Developmental changes in eyeblink conditioning and neuronal activity in the pontine nuclei. Learn. Mem. 10: 337-345.

Freeman, J.H. and Rabinak, C.A. 2004. Eyeblink conditioning in rats using pontine stimulation as a conditioned stimulus. Int. Physiol. Behav. Sci. 39: 180-191.

Freeman, J.H., Rabinak, C.A., and Campolattaro, M.M. 2005. Pontine stimulation overcomes developmental limitations in the neural mechanisms of eyeblink conditioning. Learn. Mem. 12: 255-259.

Gabriel, M., Saltwick, S.E., and Miller, J.D. 1975. Conditioning and reversal of short-latency multiple-unit responses in the rabbit medial geniculate nucleus. Science 189: 1108-1109.

Gould, T.J., Sears, L.L., and Steinmetz, J.E. 1993. Possible CS and US pathways for rabbit classical eyelid conditioning: Electrophysiological evidence for projections from the pontine nuclei and inferior olive to cerebellar cortex and nuclei. Behav. Neural Biol. 60: 172-185.
Halverson, H.E. and Freeman, J.H. 2006. Medial auditory thalamic nuclei are necessary for eyeblink conditioning. Behav. Neurosci. 120: $880-887$.

Hesslow, G., Svensson, P., and Ivarsson, M. 1999. Learned movements elicited by direct stimulation of cerebellar mossy fiber afferents. Neuron 24: 179-185.

Kawamura, K. 1975. The pontine projection from the inferior colliculus in the cat. An experimental anatomical study. Brain Res. 95: 309-322.

Knowlton, B.J. and Thompson, R.F. 1988. Microinjections of local anesthetic into the pontine nuclei reduce the amplitude of the classically conditioned eyelid response. Physiol. Behav. 43: 855-857.

Knowlton, B.J. and Thompson, R.F. 1992. Conditioning using a cerebral cortical conditioned stimulus is dependent on the cerebellum and brain stem circuitry. Behav. Neurosci. 106: 509-517.

Koutalidis, O., Foster, A., and Weisz, D.J. 1988. Parallel pathways can conduct visual CS information during classical conditioning of the NM response. J. Neurosci. 8: 417-427.

LeDoux, J.E., Ruggiero, D.A., Forest, R., Stornetta, R., and Reis, D.J. 1987. Topographical organization of convergent projections to the thalamus from the inferior colliculus and spinal cord of the rat. $J$. Comp. Neurol. 264: 123-146.

LeDoux, J.E., Iwata, J., Cicchetti, P., and Reis, D.J. 1988. Different projections of the central amygdaloid nucleus mediate autonomic and behavioral correlates of conditioned fear. I. Neurosci. 8: $2517-2529$

Lee, T. and Kim, J.J. 2004. Differential effects of cerebellar. Amaygdalar, and hippocampal lesions on classical eyeblink conditioning in rats. J. Neurosci. 24: 3242-3250.

Legg, C.R., Mercier, B., and Glickstein, M. 1989. Corticopontine projrction in the rat: The distribution of labeled cortical cells after large injections of horseradish peroxidase in the pontine nuclei. $J$. Comp. Neurol. 286: 427-441.

Lennartz, R.C. and Weinberger, N.M. 1992. Frequency-specific receptive field plasticity in the medial geniculate body induced by Pavlovian fear conditioning is expressed in the anesthetized brain. Behav. Neurosci. 106: 484-497.

Lewis, J.L., LoTurco, J.J., and Solomon, P.R. 1987. Lesions of the middle cerebellar peduncle disrupt acquisition and retention of the rabbit's classically conditioned nictitating membrane response. Behav. Neurosci. 101: 151-157.

Maren, S., Yap, S.A., and Goosens, K.A. 2001. The amygdala is essential for the development of neuronal plasticity in the medial geniculate nucleus during auditory fear conditioning in rats. J. Neurosci. 21: $1-6$.

McCormick, D.A., Lavond, D.G., and Thompson, R.F. 1983. Neuronal responses of the rabbit brainstem during performance of the classically conditioned nictitating membrane (NM)/eyelid response. Brain Res. 271: 73-88.

McEchron, M.D., McCabe, P.M., Green, E.J., Llabre, M.M., and Schneiderman, N. 1995. Simultaneous single unit recording in the medial nucleus of the medial geniculate nucleus and amygdaloid central nucleus throughout habituation, acquisition, and extinction of the rabbit's classically conditioned heart rate. Brain Res. 682: $157-166$.

Medina, J.F., Nores, W.L., and Mauk, M.D. 2002. Inhibition of climbing fibres is a signal for the extinction of conditioned eyelid responses. Nature 416: 330-333.

Nolan, B.C. and Freeman, J.H. 2006. Purkinje cell loss by OX7-saporin impairs acquisition and extinction of eyeblink conditioning. Learn. Mem. 13: 359-365.

Nowak, A.J., Kehoe, E.J., Macrae, M., and Gormezano, I. 1999. Conditioning and reflex modification of the rabbit nictitating membrane response using electrical stimulation in auditory nuclei. Behav. Brain Res. 105: 189-198.

Ohyama, T., Nores, W.L., Medina, J.F., Riusech, F.A., and Mauk, M.D. 2006. Learning-induced plasticity in deep cerebellar nucleus. $J$. Neurosci. 26: 12656-12663.

O'Connor, K.N., Allison, T.L., Rosenfield, M.E., and Moore, J.W. 1997. Neural activity in the medial geniculate nucleus during auditory trace conditioning. Exp. Brain Res. 113: 534-556.

Paxinos, G. and Watson, C. 1998. The rat brain in stereotaxic coordinates. Academic Press, New York.

Poremba, A. and Gabriel, M. 2001. Amygdalar efferents initiate auditory thalamic discriminative training-induced neuronal activity. $J$. Neurosci. 21: 270-278.

Ryugo, D.K. and Weinberger, N.M. 1978. Differential plasticity of morphologically distinct neuron populations in the medial geniculate body of the cat during classical conditioning. Behav. Biol. 22: 275-301.

Steinmetz, J.E. 1990. Neuronal activity in the rabbit interpositus nucleus during classical NM-conditioning with a 
pontine-nucleus-stimulation CS. Psychol. Sci. 1: 378-382.

Steinmetz, J.E. and Sengelaub, D.R. 1992. Possible conditioned stimulus pathway for classical eyelid conditioning in rabbits. Behav. Neural Biol. 57: 103-115.

Steinmetz, J.E., Rosen, D.J., Chapman, P.F., Lavond, D.G., and Thompson, R.F. 1986. Classical conditioning of the rabbit eyelid response with a mossy fiber stimulation CS. I. Pontine nuclei and middle cerebellar peduncle stimulation. Behav. Neurosci. 100: $878-887$.

Steinmetz, J.E., Logan, C.G., Rosen, D.J., Thompson, J.K., Lavond, D.G. and Thompson, R.F. 1987. Initial localization of the acoustic conditioned stimulus projection system to the cerebellum essential for classical eyelid conditioning. Proc. Natl. Acad. Sci. 84: 3531-3535.

Steinmetz, J.E., Lavond, D.G., and Thompson, R.F. 1989. Classical conditioning in rabbits using pontine nucleus stimulation as a conditioned stimulus and inferior olive stimulation as an unconditioned stimulus. Synapse 3: 225-233.

Supple Jr., W.F. and Kapp, B.S. 1989. Response characteristics of neurons in the medial component of the medial geniculate nucleus during Pavolvian differential fear conditioning in rabbits. Behav. Neurosci. 6: $1276-1286$

Thompson, R.F. 2005. In search of memory traces. Annu. Rev. Psychol. 56: $1-23$.

Tracy, J.A., Thompson, J.K., Krupa, D.J., and Thompson, R.F. 1998. Evidence of plasticity in the pontocerebellar conditioned stimulus pathway during classical conditioning of the eyeblink response in the rabbit. Behav. Neurosci. 112: 267-285.

Winer, J.A. 1992. The functional architecture of the medial geniculate body and the primary auditory cortex. In The mammalian auditory pathway: Neuroanatomy, Vol. 1, Springer Handbook of Auditory Research (eds. A.N. Popper, et al.), pp. 222-409. Springer-Verlag, New York.

Received November 8, 2006; accepted in revised form January 23, 2007. 


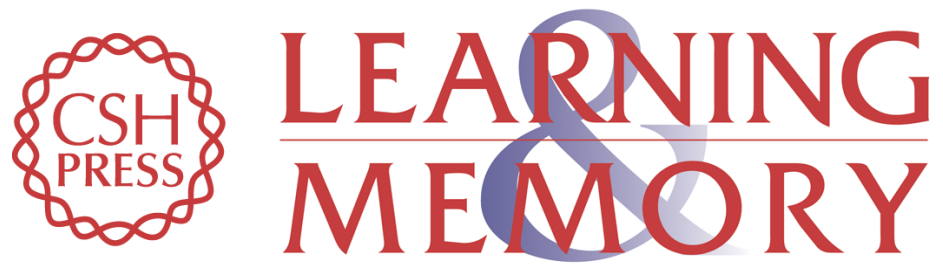

\section{Medial auditory thalamic stimulation as a conditioned stimulus for eyeblink conditioning in rats}

Matthew M. Campolattaro, Hunter E. Halverson and John H. Freeman

Learn. Mem. 2007, 14:

Access the most recent version at doi:10.1101//m.465507

References

This article cites 43 articles, 12 of which can be accessed free at:

http://learnmem.cshlp.org/content/14/3/152.full.html\#ref-list-1

License

Email Alerting

Service
Receive free email alerts when new articles cite this article - sign up in the box at the top right corner of the article or click here. 